\title{
Study on Character Recognition Arithmetic Based on Intelligent Information Processing Technologies
}

\author{
Yingyong Zou ${ }^{1,2}$, Yongde Zhang ${ }^{1}$, Xin $\mathrm{Wang}^{2}$ and Guangbin $\mathrm{Yu}^{1}$ \\ ${ }^{1}$ School of Mechanical and Power Engineering, Harbin University of Science and \\ Technology, Harbin 150080, China \\ ${ }^{2}$ Mechanical Engineering College, Changchun University, Changchun 130022, China \\ yyzou@126.com,zhangyd@hrbust.edu.cn
}

\begin{abstract}
Aiming at the complexity and limitations of traditional character recognition design method, an algorithm combined with genetic algorithms and neural network is proposed. Using this method, the advantages genetic algorithm which global optimal solution or a very good performance suboptimal solutions can easily be obtained is fully utilized. The shortcomings of neural network model such as slow convergence speed, entrapment in local optimum, unstable network structure etc are solved. Combined neural network and genetic algorithm is to make full use of the advantages of both, so that the new algorithms both neural network learning capability and robustness, but also a strong genetic algorithms global random search capability, the neural network has self-evolutionary, adaptive capacity, so as to construct evolutionary neural network. The actual application in character recognition results show that, compared with the traditional method, this model has a strong feasibility and effectiveness.
\end{abstract}

Keywords: Character recognition, Information Processing Digital image processing

\section{Introduction}

The neural network is a parallel distributed processor has a large number of connections. It has the ability to acquire knowledge and problem-solving through learning. The characteristics of the mode can be effectively extracted through trained network. The existing heuristic pattern recognition system can not be a good solution to the invariant detection, adaptive, abstract or summary can be solved [1]. But the BP neural network training is slow. It is easy to fall into the shortcomings of weak local minima and the global search ability. However, the genetic algorithm is easy to get the global optimal solution or the good performance of the suboptimal solutions. To combine neural networks and genetic algorithms, you can make full use of the advantages of both, so that the learning ability and robustness of the new algorithm is a neural network and genetic algorithm a strong global random search capabilities, making the neural network has self-evolutionary, adaptive capacity, to construct evolutionary neural network. Basic genetic algorithm to optimize the production of the initial population is random, often resulting in adaptation to spend large individual appears. With the selection, crossover and mutation operation, due to the presence of large individual, thereby increasing the probability that the individual is selected to copy, thus causing many of the offspring of the individual's ancestors are large individuals, this is the genetic algorithm, premature convergence phenomenon [2]. At this time, individuals tend to be concentrated in a region of the search space, so easily lead to convergence to a local extreme point, or if the problem exists more than one extreme point can only be searched part 
of the extreme points. In order to ensure the global convergence of the genetic algorithm to prevent the occurrence of premature convergence phenomenon, it must maintain individual diversity in the population, to avoid high-adapt to the degree of individual loss. Niche technology provides a new way to solve this problem. Niche genetic algorithm can guarantee to maintain relatively stable population diversity in the evolutionary process, in large measure to improve the ability of global optimization of the basic genetic algorithm, to avoid falling into local optimal solutions.

In this paper, the combination of niche genetic optimization algorithm and neural network is applied to the identification of mathematical symbols, not only to overcome the genetic algorithm does not have the global convergence and artificial neural network is easily trapped into local minimum point defects, but also make full use of their respective advantages. This method to extract characters complementary characteristics of the coarse grid, the projection characteristics, cross-sectional characteristics and structural characteristics, and these four features constitute the feature vector input of BP neural network fusion classification. Using a genetic algorithm optimized artificial neural network weights and thresholds, and then uses the trained NGA-BP network identification of mathematical symbols. The experimental results show that: the method has higher recognition rate and reliability.

\section{Handwritten Digit Symbol Image Preprocessing}

Handwritten mathematical symbol recognition, the character will be written on paper, by photoelectric scanning analog signals, analog to digital conversion with a gray value digital signal input into the computer. The shaped distortion, smear, broken pens, cross-linked will be caused because paper thickness, whiteness, smoothness, ink shades, printing or writing quality and other factors [3]. In addition, due to the differential rate of input devices, linearity, quantization process will generate noise, so pretreatment prior to character recognition [4]. The pretreatment generally include normalization, binarization, denoising, and refinement.

Character normalization is to eliminate the character dot matrix on the size of the deviation, to identify the characters have the same size and shape, in order to facilitate the feature extraction and recognition. Image after size normalization and position normalization is illustrated in Figure 2.1. Character binary is that digital gray scale image processing into a binary image to reduce the data storage capacity, reducing the complexity of subsequent processing. Image binarization before and after comparison is illustrated in Figure 2.2. The purpose of the character of denoising is to remove isolated noise, interference, smooth stroke edge. Effect diagram of median filtering is illustrated in Figure 2.3. Refinement in order to approximate the shape of the original region consists of a simple curve graphics. Refine the text image not only retains the original text the vast majority of features, feature extraction, and storage than the original text value of the lattice is much less, reducing the processing workload. The character refinement image is illustrated in Figure 2.4.

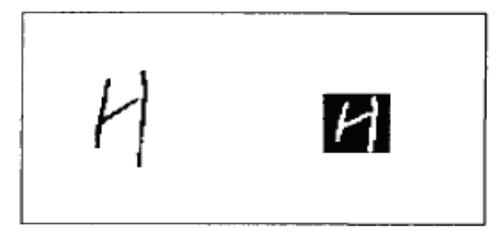

Figure 2.1. Image Normalization

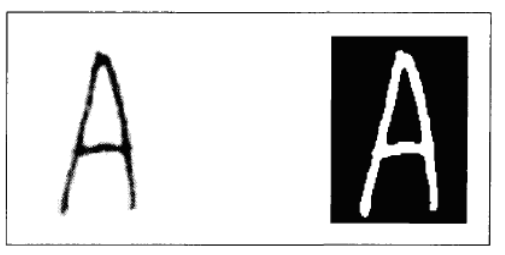

Figure 2.2. Image Binarization 


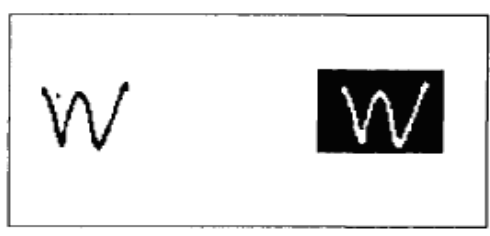

Figure 2.3. Effect Diagram of Median Filtering

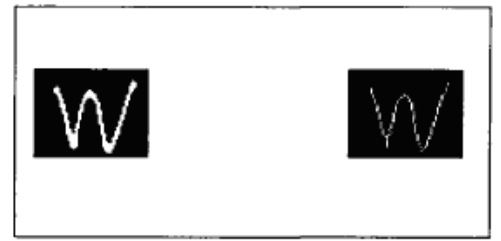

Figure 2.4. Character Refinement

\section{Character Feature Extraction}

Character features are extracted using a combination of methods of statistical characteristics and structural features to obtain additional target identification information, and help to improve the positive recognition rate and reliability of the system.

\subsection{Cross-cut Features}

Through the analysis of the characters, the main features of the character concentrated in the middle part of the picture, and the middle of the gesture changes most of the site, if you want to accurately identify the symbols, it is necessary to maximize the extraction of symbols can distinguish between the characteristics. In this paper a non-uniform cross-sectional feature extraction approach has been adopted, more to the picture the more dense central method to extract the cross-sectional characteristics. The specific method is refined image lattice horizontal divided into four parts: Images were taken, line 8, line 12, line 16, line 20, line 24 and 8, column 12, 16, 20,24 as a characteristic extraction line, its the horizontal scan can be five transverse cross-sectional and five longitudinal cross-sectional, as the eigenvectors of the crosssectional characteristics.

\subsection{Projection Characteristics}

The number of black pixels and the number of black pixels of each row, each column using image projection statistics in order to better reflect the pixel distribution. In this paper, a method of combining the vertical projection and horizontal projection, vertical projection and horizontal projection of the refined image, to form a feature vector based on the projection method.

\subsection{Structural o f Feature}

The main extract some special points in the symbols skeleton graphics, such as endpoints, discount points, trigeminal point and quad point. 


\subsection{Coarse Grid Characteristics}

First, the square lattice $(32 \times 32)$ divided into 16 small cells, each cell are $(8 \times 8)$ lattice. Each cell, from left to right, top to bottom, progressive scan, statistical points laterally within each cell is ' 1 ' appears.

\section{Character Feature Extraction}

\subsection{Determine the Network Layers}

Although in some cases, with two hidden layer neural network may bring additional benefits, but the increase in the number of hidden layer makes the network more complex, increase the computing workload and will require more samples number, so that the network's training cycle is longer [5]. Symbols identify the problem; with one hidden layer neural network has enough.

\subsection{Input and Output Mode}

The role of the buffer memory from the input layer, it receives the external input data, the number of nodes depends on the dimension of the input vector, after a symbolic feature extraction, with the feature vector as input of neural network. This article will include numbers and letters, including the commonly used 112 mathematical symbols for the study, each symbol corresponds to the feature vector length of 93, each type of symbol training for 20 groups, so the input feature matrix [2240*93].

The output layer nodes depend on two aspects of the output data type and indicate that the type size of the data required. Too much because the number of characters using 8 binary coding method to encode the symbol [00000000], for every increase of 1 , corresponding to a mathematical symbol. When the input-output model to determine the input layer and output layer of BP network corresponding to the number of neurons along OK, the input layer neurons, 93 output layer neuron number is 8 .

\subsection{BP Network Learning Rates and Learning Algorithms}

Large learning rate may lead to system instability and lead to longer training time is too small learning rate, learning rate selected is generally in the range of 0.01 to 0.8 . In this paper Select the learning rate is 0.05 and BP neural network learning algorithm is the momentum BP algorithm.

\subsection{Activation Function}

In order to improve the range of the input vector, and make the range of the output vector within the desired $(0,1)$ range, select tansig function as the activation function of hidden layer neurons, select logsig function as the output layer neuron activation function.

$$
\begin{aligned}
& \text { Tansig function: } f(x)=\frac{1-\exp (-x)}{1+\exp (-x)} \\
& \text { Logsig function: } f(x)=\frac{1}{1+\exp (-x)}
\end{aligned}
$$




\subsection{Hidden Layer Nodes}

The number of hidden layer nodes affects the accuracy of the network's training model [6]. Number of hidden nodes depends on the complexity of the law contains a select number of samples the number and size of the sample noise and sample. Implied more layer nodes, the stronger the model approximation ability, but too much of hidden nodes increases the estimation error of the model, while in the case of a limited number of samples are also prone to over fitting phenomenon, reduce network generalization ability; of hidden nodes is too small, will lead to lack of network learning ability. Therefore, considering the number of iterations and the network generalization, select the hidden layer nodes. First, according to the empirical Formula 3, calculate the implicit layer node number of rough estimates, the initial number of hidden nodes in the range of 10 to 20. Select the hidden layer nodes in order to better determine the network structure, between 10 to 20 .

$$
H=\sqrt{m+n}+a
$$

Where $\mathrm{H}$ is the hidden layer nodes, $\mathrm{m}$ is the input layer nodes, $\mathrm{n}$ is the output layer nodes, is a constant between 1 and 10 .

Feature extraction sample as the training set, 9 to 20 network training of the hidden layer nodes, and then another part of the sample as the test set, the trained network to test and found that when the hidden layer nodes between 16 to 20, the network convergence steps, considering the convergence speed and recognition rate, the hidden layer nodes is determined to be 20 .

\section{Training and Recognition of the Network Model}

After the NGA-BP mathematical symbol recognition model is established, the network training starts and comparative analysis of training results with the original $\mathrm{BP}$ model.

Table 5.1. The NGA-BP and BP Model Convergence Comprehensive Tables

\begin{tabular}{c|c|c}
\hline $\begin{array}{c}\text { Training } \\
\text { methods }\end{array}$ & $\begin{array}{c}\text { Average convergence } \\
\text { steps }\end{array}$ & $\begin{array}{c}\text { the number of non } \\
\text { convergence }\end{array}$ \\
\hline NGA-BP model & 27643.2 & 5 \\
\hline BP model & 36472.6 & 14 \\
\hline
\end{tabular}

Statistics in the table show that for the same training methods, the convergence rate of the two network training have great differences. NGA-BP algorithm improves the convergence speed and convergence stability. The NGA-BP network model established in this paper the data samples for training and recognition, the recognition results are shown in Table 5.2.

Table 5.2. Recognition Results Statistical Table

\begin{tabular}{c|c|c|c}
\hline Test methods & $\begin{array}{c}\text { Correctly } \\
\text { recognition }\end{array}$ & Error recognition & Recognition rate \\
\hline NGA-BP model & 906 & 94 & $90.6 \%$ \\
\hline BP model & 784 & 216 & $78.4 \%$ \\
\hline
\end{tabular}




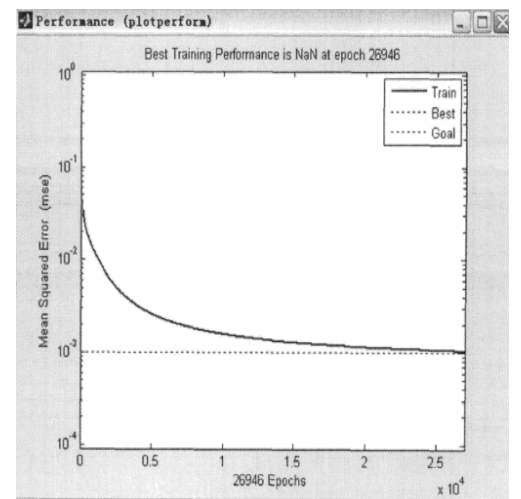

Figure 5.1. NGA-BP Algorithm

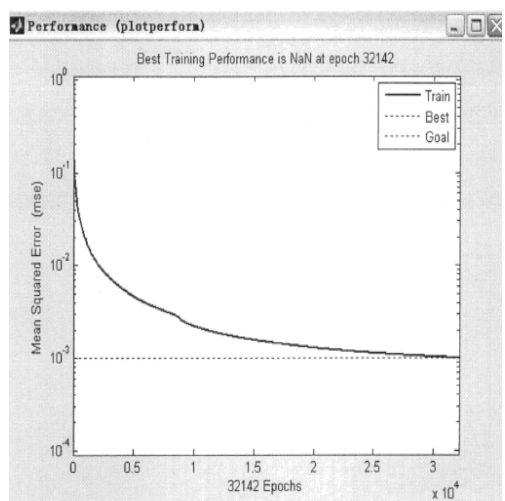

Figure 5.2. BP Algorithm

\section{Figure 5.1. Is the Convergence Curve of the NGA-BP Algorithm, Fig.5.2 is the Convergence Curve of the BP Algorithm}

Experiments show that the convergence rate of the NGA-BP network model was $21.7 \%$ higher than the original BP model. The NGA-BP network model of recognition rate is 12.2 percent higher than the original $\mathrm{BP}$ model. Visible, the overall performance of the NGA-BP network model is superior to the BP neural network model to improve the recognition accuracy of the mathematical symbols.

\section{Conclusion}

The genetic algorithm is highly efficient, parallel, strong robustness and adaptive advantages, can solve complex optimization problems. The artificial neural network with adaptive self-organization, strong learning, association and fault tolerance, can be a good solution to uncertain and ambiguous, complex information processing problems. This paper will combine the advantages of both, and overcome through improved niche genetic algorithm neural network convergence is slow and likely to fall into local minimum point defects, and genetic algorithm may occur in the population is unevenly distributed premature convergence into local minima defects effectively solve the uncertain, ambiguous, complex handwritten mathematical character recognition.

\section{Acknowledgements}

The research is carried out in The Innovation Center of Disabled Rehabilitation in Equipment and Technology is supported by Jilin Provincial Science and Technology Department (No. 20102109). The research activities have also been funded by Changchun Science and Technology (No. 10PT01), and Key Program of National Natural Science Foundation of Heilongjiang No.ZD201309.

\section{References}

[1] M.S. ZhijunLiu, "A genetic algorithm approach used to generate the network structures", Proceedings of the 1999 IEEE/RSJ International conference on intelligent Robots and System, Kyongju, SouthKorea, vol. 1, (1999), pp.763-768.

[2] L. LiMingqiang and K. Jisong, "Effective method of preventing premature of genetic algorithm", Transaction of Tianjin University, vol. 5, no. 1, (1999), pp. 31-35. (in Chinese)

[3] Y. Tao and Y.Y. Tang, "The Feature Extraction of Chinese Character Based On Contour Information”, ICDAR vol. 99, (1999) September, pp. 637-640. 
[4] C.J.M. Ahmadi and M. Shridhar, "Recongnition of handwritten numerals with mu1tiple feature and multistage classifier", Pattern Recongnition, (in Chinese), vol. 28, no. 2, (1995), pp. 153-160.

[5] J.S.Fan, Q.Tao and T.J.Fang, "Genetic Algorithm optimizes Neural Network Based on Structure Risk Minimization", Proceedings of 3rd word congress on Intelligent Control and Automation, (2000) June 28-July 2, pp. 948-952.

[6] M. Srinvivas and L. M. Patnail, "Adaptive Probabilities of Crossover and Mutation in Genetic Algorithms”, IEEE Trans. On Systems, Man and Cybernetics, vol. 24, no. 4, (1994), pp. 656-667. 
International Journal of Signal Processing, Image Processing and Pattern Recognition Vol.7, No.3 (2014) 